\title{
Identification of a Novel DFR-A Mutant Allele Determining the Bulb Color Difference between Red and Yellow Onions (Allium cepa L.)
}

\author{
Bongju Kim, Youngcho $\mathrm{Cho}^{2}$, Sunggil Kim ${ }^{1} *$ \\ ${ }^{1}$ Department of Plant Biotechnology, Biotechnology Research Institute, Chonnam National University, Gwangju 61186, Korea \\ ${ }^{2}$ ONBREETECH Corp., Haenam 59049, Korea
}

\begin{abstract}
To introduce downy mildew resistance from a yellow-colored resistant cultivar, 'Santero', into a yellow breeding line, OT803, the $F_{1}$ hybrid was produced by crossing Santero and OT803. The bulb color of the $\mathrm{F}_{1}$ hybrids became light pink, suggesting involvement of complementation between the $D F R-A$ and $A N S$ genes in the onion anthocyanin biosynthesis pathway. Since Santero contained active $D F R-A$ and inactive $A N S$ alleles, OT803 was assumed to harbor active $A N S$ and inactive $D F R-A$ alleles. However, some yellow-colored individuals of OT803 were shown to contain the homozygous genotype of the active $D F R-A^{R 4}$-like allele. Sequencing of 4,830-bp full-length sequences of this $D F R-A^{R 4}$-like allele revealed that the nucleotide sequences of the $D F R-A^{R 4}$ and $D F R-A^{R 4}$-like alleles were identical except for a single nucleotide deletion in the last exon. This single base-pair deletion resulted in creation of a premature stop codon at 2-bp downstream of the deletion mutation. This new DFR- $A$ mutant allele was designated $D F R-A^{P S 2}$. The RT-PCR results showed that transcripts of the $D F R-A^{P S 2}$ allele were significantly reduced, suggesting involvement of nonsense-mediated mRNA decay (NMD) mechanism. The systematic process consisting of PCR amplification and sequencing of the PCR products was modified to identify the $D F R-A^{P S 2}$ allele among 16 different $D F R-A$ alleles. No additional accession was found to contain the $D F R-A^{P S 2}$ allele from 155 diverse onion germplasm, indicating very limited distribution of this new $D F R-A^{P S 2}$ allele.
\end{abstract}

Keywords Onion, Allium cepa, Bulb color, Dihydroflavonol 4-reductase (DFR), Molecular marker

\section{INTRODUCTION}

Bulb color is one of major traits in onion (Allium cepa L.) breeding programs (Brewster 1994), and diverse bulb colors such as red, yellow, white, and chartreuse have been reported (El-Shafie and Davis 1967). Flavonoid compounds are known to confer bulb colors in onions (Fossen et al. 1996). Flavonoid is one of major secondary metabolites, and more than 8,000 derivatives have been reported in plant species (Veitch and Grayer 2011). Flavonoid plays such diverse roles as protectants against UV radiation and pathogens in plants (Shirley 1996; Fini et al. 2011). In addition, as human diet, it has health-pro- moting effects as potent antioxidant and anticancer agents (Cook and Samman 1996; Lotito and Frei 2006; Clere et al. 2011; Cushnie and Lamb 2011; Nishiumi et al. 2011; Russo et al. 2012).

Anthocyanin, one of major flavonoid compounds, is responsible for the red bulb color, and quercetin derivatives are predominant in yellow onions (Fossen et al. 1996; Rhodes and Price 1996; Slimestad et al. 2007). Inheritance studies (Reiman 1931; Clarke et al. 1944; El-Shafie and Davis 1967) reported that five major loci were involved in determination of onion bulb colors. The $I$ locus, known as a color inhibiting factor, determines the white bulb color when its genotype is homozygous dominant. Meanwhile,

Received January 4, 2017; Revised January 26, 2017; Accepted January 26, 2017; Published March 1, 2017

*Corresponding author Sunggil Kim, dronion@jnu.ac.kr, Tel: +82-62-530-2061, Fax: +82-62-530-2069 
the $C$ locus, known as a basic color factor, also determines the white bulb color only when its genotype is homozygous recessive. When the genotype of the $G$ locus is homozygous recessive, the chartreuse bulb color appears. The $R$ and $L$ loci are complementarily involved in determination of color difference between yellow and red bulb colors.

These five loci was assumed to be related with genes involved in anthocyanin biosynthesis pathway (Kim et al. 2004a). Structural genes encoding enzymes in the anthocyanin biosynthesis pathway have been well characterized in many plant species (Goodrich et al. 1992; Quattrocchio et al. 1993; Holton and Cornish 1995; Spelt et al. 2000; Yamazaki et al. 2003). In addition, regulatory genes controlling transcription of structural genes in the pathway have also been isolated in some model species such as Arabidopsis, petunia, and maize. The ternary complex consisting of R2R3-MYB, bHLH, and WD40 transcription factors is commonly identified as major regulatory genes (Ramsay and Glover 2005; Petroni and Tonelli 2011; Czemmel et al. 2012).

The $R$ and $L$ loci were revealed to encode dihydroflavonol 4-reductase (DFR) and anthocyanidin synthase (ANS) enzymes, respectively, in the onion anthocyanin biosynthesis pathway (Kim et al. 2004a, 2005a). One leaky and three inactive $A N S$ alleles were identified from diverse onion germplasm (Kim et al. 2004b, 2005a, 2016). Recently, integration of a non-autonomous DNA transposon in the promoter region was revealed to cause significant reduction of the $A N S$ gene transcription (Kim et al. 2015). Three homologous genes coding for the DFR enzyme were isolated in the previous study (Kim et al. 2005b), and they were designated $D F R-A, D F R-B$, and $D F R-C$. Among them, only the $D F R-A$ gene was active and determined the bulb color phenotypes (Kim et al. 2005b). Since the first inactive $D F R-A$ allele was identified (Kim et al. 2005b), eleven mutant alleles containing diverse insertion, deletion, or substitution mutations have been identified (Kim et al. 2009, 2015; Song et al. 2014). Three of them harbored transposable elements including a LTR retrotransposon and two different DNA transposons (Kim et al. 2015).

In this study, a novel $D F R$ - $A$ allele containing a premature stop codon, which was created by one base-pair deletion, was identified from one breeding line. The inactivation mechanism of this mutant allele was proposed, and a systematic process for identification of 16 different $D F R-A$ alleles was updated to include the newly identified $D F R-A$ allele.

\section{MATERIALS AND METHODS}

\section{Plant materials}

A $F_{1}$ population originating from the cross between a downy mildew resistant cultivar, 'Santero', and a breeding line (OT803) was used to identify a new $D F R-A$ allele. To assess the frequency of the new $D F R-A$ allele, total genomic DNAs of 18 accessions containing the $D F R-A^{R 4}$ allele were analyzed (Supplementary Table 1). These 18 accessions were selected from 155 accessions collected from diverse countries in the previous study (Song et al. 2014). Individual plants of OT803 which contained the homozygous genotype of the new mutant $D F R-A$ allele and a red-colored breeding line, H6, were used for RT-PCR amplification of the $D F R-A$ gene.

\section{DNA extraction, PCR amplification, and sequencing of PCR products}

Total genomic DNAs were extracted from leaves of seedling or sprouted bulbs using a cetyl trimethylammonium bromide (CTAB) method (Doyle and Doyle 1987). PCR was performed in $25-\mu \mathrm{L}$ reaction mixtures containing $0.05 \mu \mathrm{g}$ template, $2.5 \mu \mathrm{L} 1 \times$ PCR buffer, $0.2 \mu \mathrm{L}$ forward primer $(10 \mu \mathrm{M}), 0.2 \mu \mathrm{L}$ reverse primer $(10 \mu \mathrm{M})$, $0.2 \mu \mathrm{L}$ dNTPs ( $10 \mathrm{mM}$ each), and $0.25 \mu \mathrm{L}$ polymerase mix (Advantage 2 Polymerase Mix; Clontech, Palo Alto, CA, U.S.A.). The primer sequences used in this study are listed in Table 1.

PCR amplification was performed with an initial denaturation step at $94^{\circ} \mathrm{C}$ for 5 minutes, followed by 40 cycles of $94^{\circ} \mathrm{C}$ for 30 seconds, $65^{\circ} \mathrm{C}$ for 30 seconds, $72^{\circ} \mathrm{C}$ for 3 minutes, and a final 10 minutes extension at $72^{\circ} \mathrm{C}$. The PCR products were visualized on a $1.5 \%$ agarose gel after ethidium bromide staining. For sequencing of PCR products, they were first purified using a QIAquick PCR Purification kit (QIAGEN, Valencia, CA, U.S.A.), and 
Table 1. Primer sequences used in this study.

\begin{tabular}{|c|c|c|c|}
\hline Name & Sequence ( $5^{\prime}$ to $\left.3^{\prime}\right)$ & Application & Reference \\
\hline DFR-F & ATGCCAGTGGAGTGCATGTTGAATGGT & $D F R-A$ allele identification & Kim et al. (2005b) \\
\hline DFR-R & TGGGTAGCGATTGGTTCATTCTCTTCA & $D F R-A$ allele identification and RT-PCR & Kim et al. (2005b) \\
\hline DFR-LR3 & TTGCAAACTCCCATGCAGCTTTCTCTG & $D F R-A$ allele identification & Song et al. (2014) \\
\hline DFR-LR4 & TTAACCATCTGCCCCACAAT & $D F R-A$ allele identification & This study \\
\hline DFR-LF5 & GAGGAGCACCAAAAGCCCGAATACGAT & RT-PCR & Song et al. (2014) \\
\hline Tubulin-F & CTGGGAGCTTTACTGCCTTG & RT-PCR & Song et al. (2014) \\
\hline Tubulin-R & CAAGGGACCTCCTGCAAATA & RT-PCR & Song et al. (2014) \\
\hline CHS-F & GAGGGTCCAGCCACGGTGTTAGCTG & RT-PCR & Kim et al. (2004b) \\
\hline CHS-R & ATCAATGGCCACACTCCTAAGCACC & RT-PCR & Kim et al. (2004b) \\
\hline F3H-F & GAAGACGAGCGTCCCAAGATTGCAT & RT-PCR & Kim et al. (2004b) \\
\hline F3H-R & TCCACCATCTTTAGTCGCTTGCAGACC & RT-PCR & Kim et al. (2004b) \\
\hline
\end{tabular}

sequencing reactions were performed by a specialized company (Macrogen, Seoul, Republic of Korea). The full-length sequences of the new $D F R-A$ allele were obtained using the method described in the previous study (Song et al. 2014).

\section{RNA extraction, cDNA synthesis, and RT-PCR}

Total RNAs were extracted from fresh leaf sheaths of four-leaf stage seedlings using a RNA extraction kit (RNeasy Plant Mini Kit, QIAGEN) following the manufacturer's instructions. cDNAs were synthesized using a commercial cDNA synthesis kit (SuperScript ${ }^{\mathrm{TM}}$ III first-strand synthesis system for RT-PCR; Invitrogen, Carlsbad, CA, U.S.A.). RT-PCR amplification was performed with an initial denaturation step at $94^{\circ} \mathrm{C}$ for 3 minutes followed by 30 cycles of $94^{\circ} \mathrm{C}$ for 30 seconds, $65^{\circ} \mathrm{C}$ for 30 seconds, and $72^{\circ} \mathrm{C}$ for 3 minutes, and a final 10 -minute extension at $72^{\circ} \mathrm{C}$. The primer pair used in RT-PCR is listed in Table 1. The onion tubulin sequence obtained from EST sequences (TC125) from the DFCI Allium cepa Gene Index (Antonescu et al. 2010) was used as a control.

\section{RESULTS}

\section{Identification of a novel $D F R$ - $\boldsymbol{A}$ mutant allele} determining the yellow bulb color in onion

In order to introduce downy mildew resistance from a resistant cultivar, Santero, into a breeding line (OT803), the $F_{1}$ hybrids were produced by the cross between Santero and

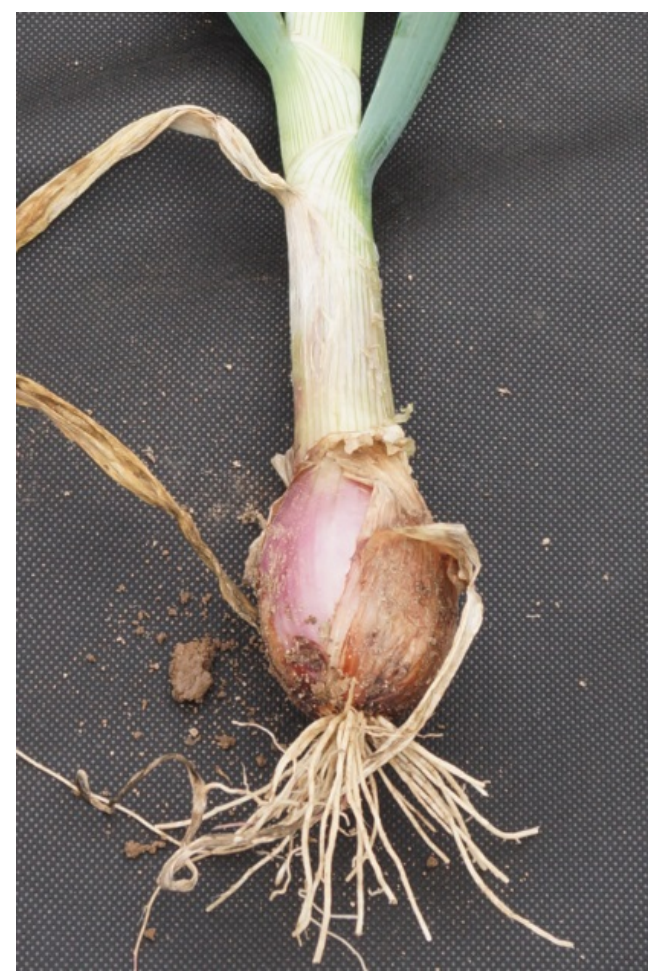

Fig. 1. The onion bulb of the $F_{1}$ hybrid between a yellow cultivar, Santero, and a yellow breeding line, OT803.

OT803. Although the bulb color of both parental lines were yellow, the bulb color of the $F_{1}$ hybrids became light pink (Fig. 1). The light pink bulb color was assumed to appear by complementation between the DFR-A and ANS genes involved in the anthocyanin biosynthesis pathway as shown in the previous study (Kim et al. 2005a). Since Santero contained an active $D F R-A^{R 3}$ allele and inactive $A N S^{P S}$ and $A N S^{S I 88 L}$ alleles (Kim et al. 2016), OT803 was 
assumed to harbor an inactive $D F R-A$ allele and an active $A N S$ allele. As expected, ten individuals of OT803 contained all active $A N S^{h l}$ allele (Table 2). Regarding the $D F R-A$ gene, the inactive $D F R-A^{T T A}$ and the active $D F R-A^{R 4}$-like alleles were identified from ten individuals, although the leaf sheath color of ten individuals were apparently yellow.

The 4,830-bp full-length sequences including putative promoter regions of the $D F R-A^{R 4}$-like allele were obtained by sequencing of the PCR products. The nucleotide sequences of the $D F R-A^{R 4}$-like allele were identical to those of the $D F R-A^{R 4}$ allele except for a single base-pair change positioned at the 121th nucleotide of the last exon (Fig. 2). The 121th cytosine nucleotide in the last exon was deleted in the $D F R-A^{R 4}$-like allele. This single base-pair deletion created a premature stop codon at 2-bp downstream of the deletion mutation (Fig. 3).

In the previous study (Kim et al. 2009), transcripts of the $D F R-A^{P S}$ allele containing a premature stop codon in the exon3 (Fig. 2) were shown to be significantly reduced. The

Table 2. Composition of $D F R-A$ and $A N S$ alleles of parental lines (Santero and OT803) and the $\mathrm{F}_{1}$ hybrid. The active $D F R-A$ and $A N S$ alleles are shown in boldfaces. The $D F R-A^{R 4}$-like allele is designated as $D F R-A^{P S 2}$ allele.

\begin{tabular}{|c|c|c|}
\hline \multirow{2}{*}{ Population } & \multicolumn{2}{|l|}{ Genotype } \\
\hline & $D F R-A$ & $A N S$ \\
\hline Santero & $D F R-A^{R 3} / D F R-A^{R 3}$ & $A N S^{P S} / A N S^{S 188 L}$ \\
\hline $\begin{array}{l}\text { OT } 803 \\
F_{1} \text { hybrid }\end{array}$ & $\begin{array}{l}\text { Mixed with homozygous and heterozygous } D F R-A^{P S 2} \text { and } D F R-A^{T T A} \\
\qquad D F R-A^{P S 2} / \mathbf{D F R}-\boldsymbol{A}^{R 3}\end{array}$ & $\begin{array}{l}A N S^{h 1} / A N S^{h 1} \\
A N S^{h 1} / A N S^{S 188 L}\end{array}$ \\
\hline
\end{tabular}
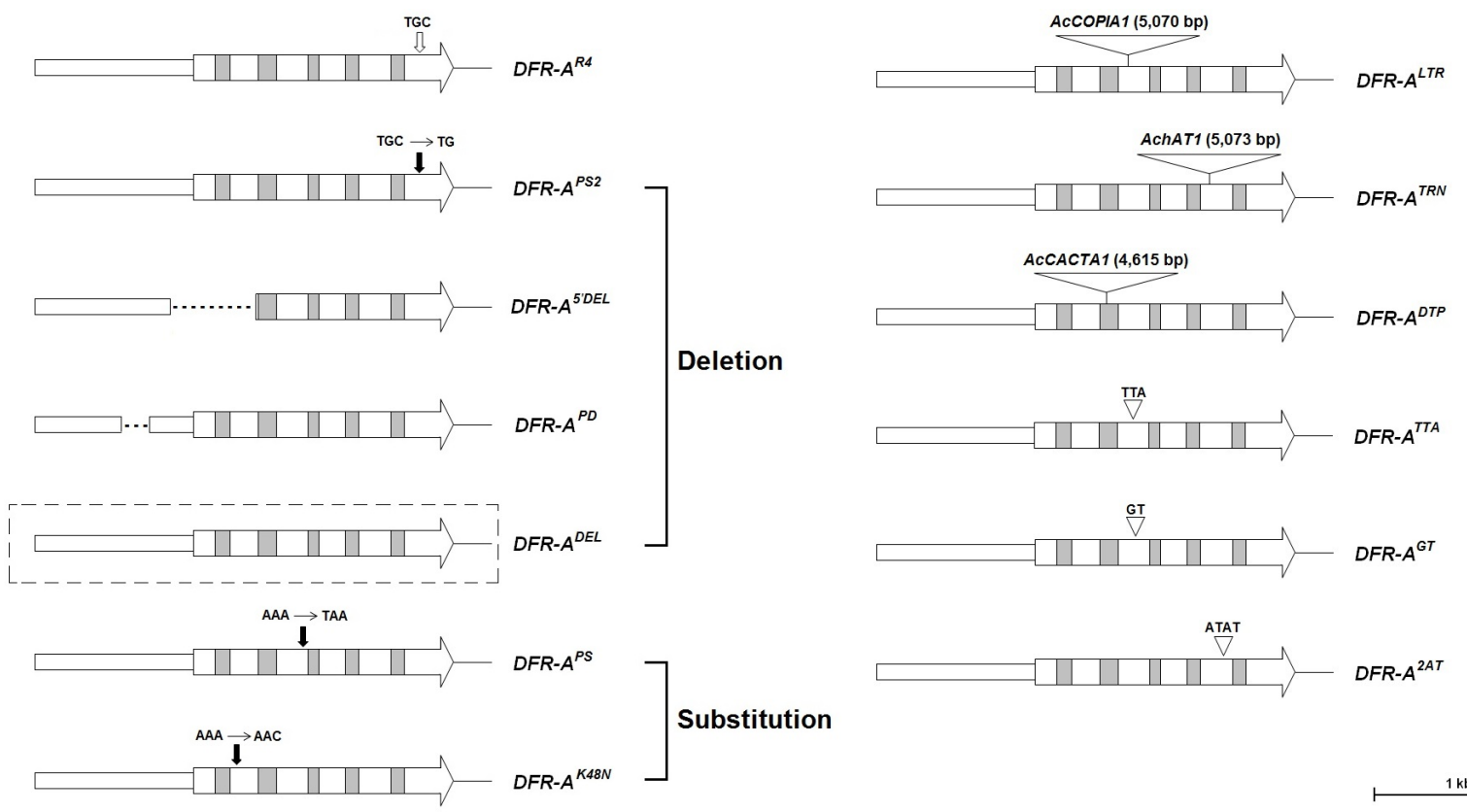

Insertion
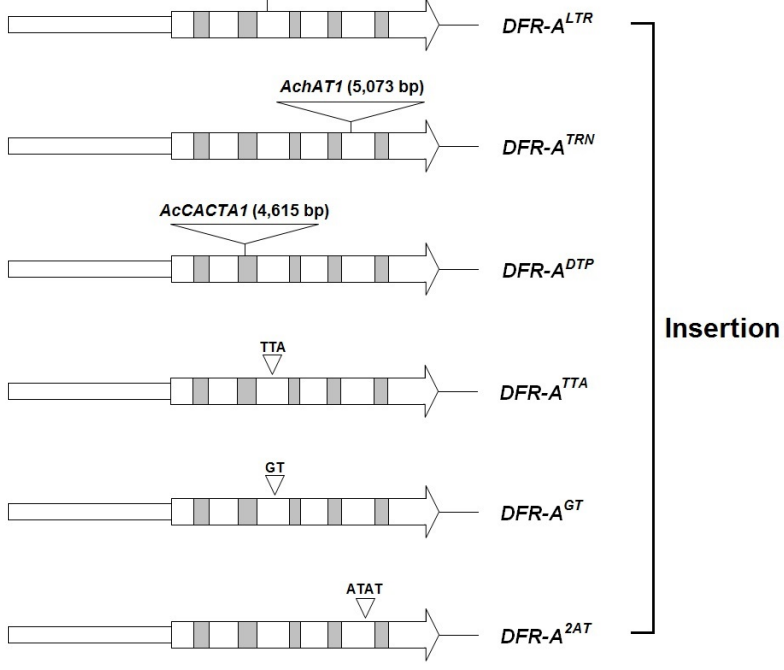

Fig. 2. Organization of the inactive mutant $D F R-A$ alleles isolated from onions. Arrow-shaped boxes indicate coding regions and the 5'-to-3' direction. The empty and gray boxes in the coding regions indicate exons and introns, respectively. The vertical filled arrows on the exons indicate the positions of sequence changes. The nucleotide sequences on the vertical empty arrow indicate the normal sequences of the functional alleles. The dotted lines in the promoter region indicate the deleted sequences. The dotted line outside the $D F R-A^{D E L}$ allele indicate entire deletion of the genic region. Nucleotide sequences and names on the inverted triangles indicate inserted sequences and transposable elements, respectively. Lengths of transposable elements are shown in parenthesis. 
Fig. 3. Alignment of partial nucleotide sequences of the $D F R-A^{R 4}$ and $D F R-A^{P S 2}$ alleles. The vertical filled arrow indicates the positon of single base-pair deletion. The rectangular box indicates the position of the premature stop codon.

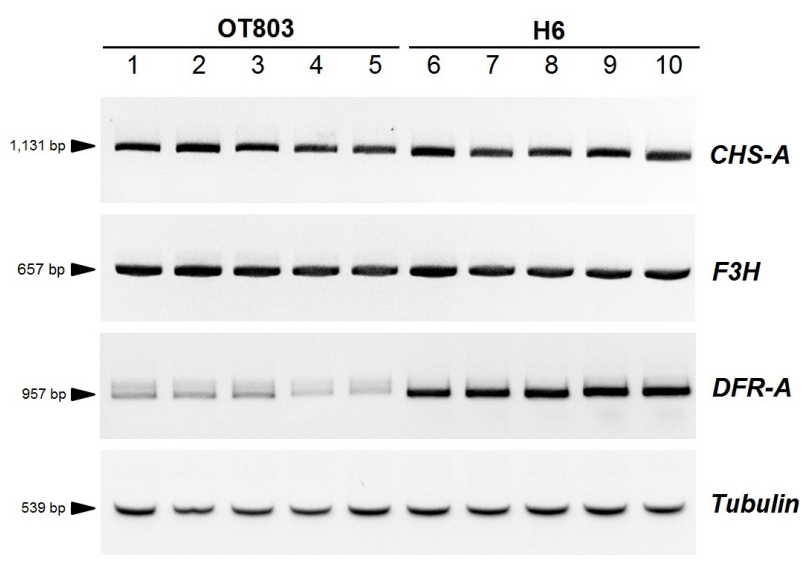

Fig. 4. RT-PCR amplification of the $D F R-A$ gene. 1-5: individuals of OT803 containing the homozygous $D F R-A^{P S 2}$ allele. 6-10: individuals of H6 containing the homozygous $D F R-A^{R I}$ allele. $C H S$ - $A$ : chalcone synthase-A, $F 3 H$ : flavanone 3-hydroxylase

nonsense-mediated mRNA decay (NMD) mechanism was proposed to cause degradation of the mRNA transcripts. To verify whether transcripts of the $D F R-A^{R 4}$-like allele were reduced by the same NMD mechanism, RT-PCR amplification was performed using five OT803 individuals harboring the homozygous $D F R-A^{R 4}$-like allele. Results showed that the $D F R-A$ transcripts of all OT803 individuals were significantly reduced, and bands of the PCR products were smear, compared with the red breeding line 'H6' which contained the active $D F R-A^{R I}$ allele (Fig. 4). However, transcription levels of other structural genes (CHS-A and $F 3 H$ ) involved in the onion anthocyanin biosynthesis pathway were not significantly different between OT803 and H6 individuals (Fig. 4). Hereafter, this novel mutant allele is designated $D F R-A^{P S 2}$.

\section{Frequency of the $D F R-A^{P S 2}$ allele in diverse onion germplasm}

For identification of specific $D F R-A$ alleles from diverse onion germplasm, a systematic process consisting of PCR amplification and sequencing of the PCR products was devised for identification of 15 different $D F R$ - $A$ alleles in the previous studies (Song et al. 2014; Kim et al. 2015). For identification of the $D F R-A^{P S 2}$ allele, one additional step of sequencing of the PCR product using the DFR-LR4 primer was added in the process to distinguish the $D F R-A^{R 4}$ and $D F R-A^{P S 2}$ alleles (Fig. 5).

In the previous study (Song et al. 2014), the DFR-A genotypes of 155 onion accessions originating from diverse countries were identified by using the process for identification of $15 D F R-A$ alleles. However, at that time, the $D F R-A^{P S 2}$ allele could not be distinguished from the $D F R-A^{R 4}$ allele by such process since the position of the 1-bp deletion in the $D F R-A^{P S 2}$ allele could not be sequenced by the DFR-LR3 primer (Fig. 5). Therefore, PCR products of 18 accessions containing the $D F R-A^{R 4}$ allele were further sequenced by the DFR-LR4 primer following the updated process (Fig. 5). However, sequencing results showed that no accession contained the $D F R-A^{P S 2}$ allele, indicating very limited distribution of the $D F R-A^{P S 2}$ allele in onion germplasm. 


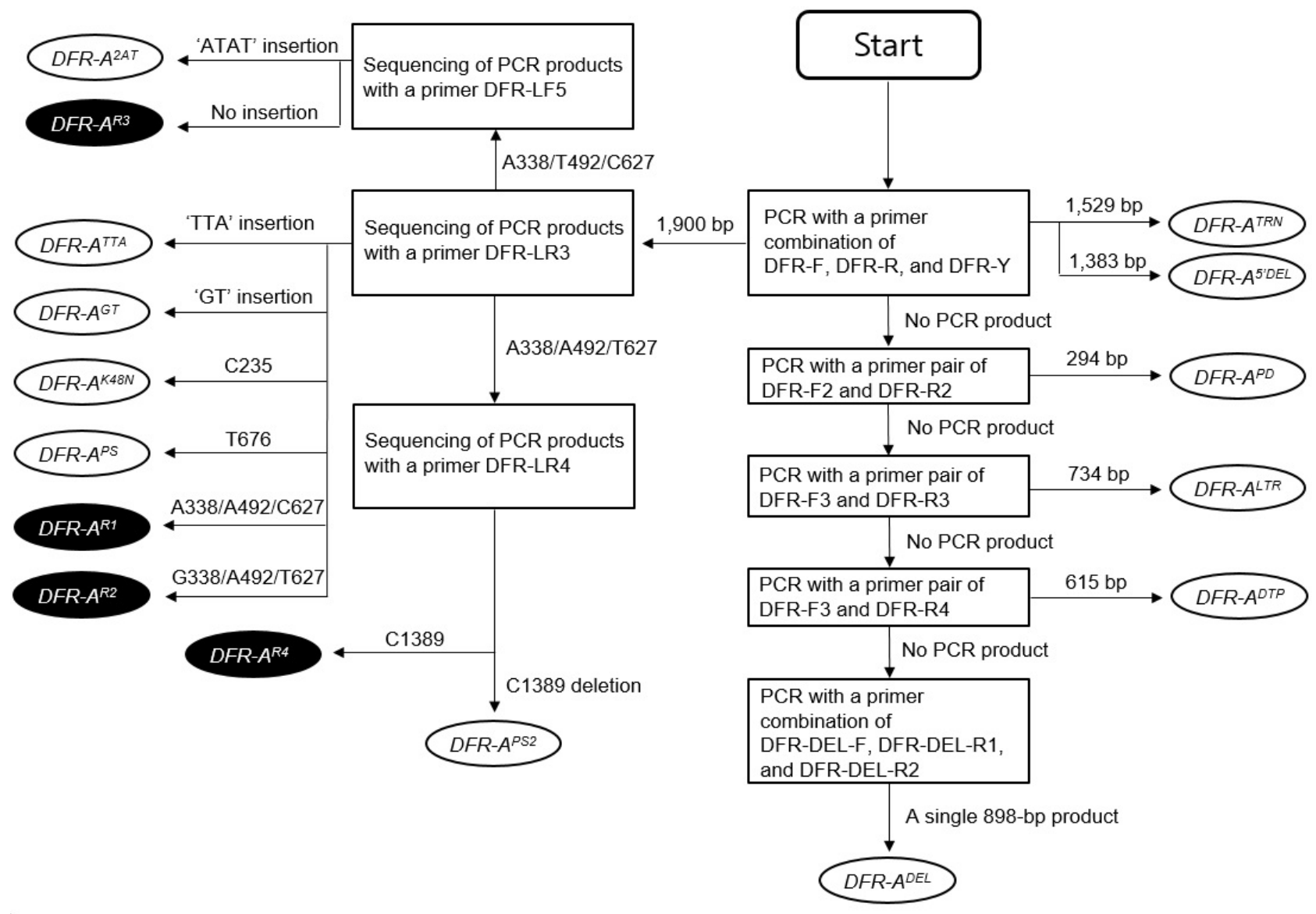

Fig. 5. Flowchart showing the process for identification of $16 D F R-A$ alleles. The SNPs are shown with numbers indicating the distances from the start codon of the DFR-A gene. Four active and 12 inactive DFR-A alleles are shown in filled and empty oval-shaped circles, respectively.

\section{DISCUSSION}

A novel inactive $D F R-A$ allele containing a premature stop codon in the last exon was identified in this study. This is the $12^{\text {th }}$ inactive $D F R-A$ allele identified from onion germplasm. In the previous studies (Kim et al. 2005b, 2009, 2015; Song et al. 2014), eleven inactive DFR-A alleles containing diverse insertion, deletion, or substitution mutations were identified (Fig. 2). Unlike other structural and regulatory genes involved in the onion anthocyanin biosynthesis pathway, the $D F R-A$ gene unusually has a large number of mutant alleles. No inactive mutant alleles of other structural and regulatory genes affecting color phenotypes have been identified in onions except for three ANS (Kim et al. 2005a, 2016) and one CHI (Kim et al. 2004c) mutant alleles. Although presence of multiple homologous $D F R$ genes in the onion genome is likely to contribute to creation of a multitude of $D F R-A$ mutants, the exact mechanism responsible for frequent mutation of the $D F R-A$ gene is not resolved yet. Khar et al. (2008) showed that numerous copies of DFR homologs existed in the onion genome by Southern blot analysis.

The $D F R-A^{P S 2}$ allele is probably derived from the $D F R-A^{R 4}$ allele recently, since there is only a single nucleotide deletion between 4,830-bp promoter and coding regions of the $D F R-A^{R 4}$ and $D F R-A^{P S 2}$ alleles. The single nucleotide deletion changes the reading frame and create a premature stop codon. However, loss of the last 60 residues, which is caused by the premature stop codon, may not have a significant effect on the function of the DFR enzyme, since the premature stop codon is positioned at the last exon. Indeed, none of the catalytic triad and 28 residues 
involved in binding of substrate and NADPH is positioned in the last exon (Petit et al. 2007).

However, the RT-PCR result showed that the transcripts of the $D F R-A^{P S 2}$ allele were significantly reduced, and the PCR products appeared as smear bands compared with the normal $D F R-A^{R I}$ allele. Since there is no polymorphism in the $2.5-\mathrm{kb}$ upstream region containing putative promoter regions between the $D F R-A^{R 4}$ and $D F R-A^{P S 2}$ alleles, transcription of the $D F R-A^{P S 2}$ allele is assumed to be normal. Therefore, the NMD mechanism is most likely to be responsible for degradation of the $D F R-A^{P S 2}$ mRNA transcripts. The NMD mechanism, which is well conserved in all eukaryote, plays an important role in preventing deleterious effects of truncated proteins by degradation of defective mRNAs harboring premature stop codons (Hentze and Kulozik 1999; Neu-Yilik et al. 2004; Chang et al. 2007; Shaul 2015).

Although the causal genes responsible for the bulb color difference between red and yellow onions have been revealed as the genes coding for DFR and ANS enzymes (Kim et al. 2004a, 2005a), designing of universal functional markers based on mutations is not a straightforward process due to a high level of allelic variation of the $D F R-A$ gene. In addition, complementation between the $D F R-A$ and $A N S$ genes makes it more complicated to identify specific $D F R-A$ alleles of yellow onion germplasm. Therefore, systematic processes are first required for identification of specific alleles of the $D F R-A$ and $A N S$ genes. Once specific $D F R-A$ or $A N S$ alleles of parental lines in segregating populations are determined, functional markers can be designed on the basis of polymorphism between two alleles. We updated the process for identifying $16 D F R$ - $A$ alleles in this study. Combined with the process for $A N S$ allele identification (Kim et al. 2016), the updated process for $D F R-A$ allele identification becomes an essential tool in onion breeding programs.

\section{ACKNOWLEDGEMENTS}

This research was supported by the Agriculture Research Center program, Golden Seed Project (Center for Horticultural Seed Development, No 213007-05-1-SBB10) and a grant from the Next-Generation BioGreen 21 Program (Plant Molecular Breeding Center No. PJ011034). The authors thank Ji-wha Hur, Jeong-Ahn Yoo, and Su-jung Kim for their dedicated technical assistance.

\section{REFERENCES}

Antonescu C, Antonescu V, Sultana R, Quackenbush J. 2010. Using the DFCI gene index databases for biological discovery. Curr. Protoc. Bioinformatics Unit1.6.1Unit1.636.

Brewster JL. 1994. Onions and other vegetable Alliums. CAB International, Wallingford, UK. pp.1-26.

Chang Y, Imam JS, Wilkinson MF. 2007. The nonsensemediated decay RNA surveillance pathway. Annu. Rev. Biochem. 76: 51-74.

Clarke AE, Jones HA, Little TM. 1944. Inheritance of bulb color in the onion. Genetics 29: 569-575.

Clere N, Faure S, Martinez MC, Andriantsitohaina R. 2011. Anticancer properties of flavonoids: roles in various stages of carcinogenesis. Cardiovasc. Hematol. Agents Med. Chem. 9: 62-77.

Cook NC, Samman S. 1996. Flavonoids- Chemistry, metabolism, cardioprotective effects, and dietary sources. Nutr. Biochem. 7: 66-76.

Cushnie TP, Lamb AJ. 2011. Recent advances in understanding the antibacterial properties of flavonoids. Int. J. Antimicrob. Agents 38: 99-107.

Czemmel S, Heppel SC, Bogs J. 2012. R2R3 MYB transcription factors: key regulators of the flavonoid biosynthetic pathway in grapevine. Protoplasma 249: S109-S118.

Doyle JJ, Doyle JL. 1987. A rapid DNA isolation procedure for small quantities of fresh leaf tissue. Phytochem. Bull. 19: 11-15.

El-Shafie MW, Davis GN. 1967. Inheritance of bulb color in the onion (Allium cepa L.). Hilgardia 38: 607-622.

Fini A, Brunetti C, Di Ferdinando M, Ferrini F, Tattini M. 2011. Stress-induced flavonoid biosynthesis and the antioxidant machinery of plants. Plant Signal Behav. 6: 709-711.

Fossen T, Andersen OM, Ovstedal DO, Pedersen AT, Raknes A. 1996. Characteristic anthocyanin pattern from onions and other Allium spp. J. Food Sci. 61: 703-706. 
Goodrich J, Carpenter R, Coen ES. 1992. A common gene regulates pigmentation pattern in diverse plant species. Cell 68: 955-964.

Hentze MW, Kulozik AE. 1999. A perfect message: RNA surveillance and nonsense-mediated decay. Cell 96: 307-310.

Holton TA, Cornish EC. 1995. Genetics and biochemistry of anthocyanin biosynthesis. Plant Cell 7: 1070-1083.

Khar A, Jakse J, Havey MJ. 2008. Segregations for onion bulb colors reveal that red is controlled by at least three loci. J. Amer. Soc. Hort. Sci. 133: 42-47.

Kim E, Kim C, Kim S. 2016. Identification of two novel mutant $A N S$ alleles responsible for inactivation of anthocyanidin synthase and failure of anthocyanin production in onion (Allium cepa L.). Euphytica 212: 427-437.

Kim S, Baek D, Cho DY, Yoon M. 2009. Identification of two novel inactive $D F R-A$ alleles responsible for failure to produce anthocyanin and development of a simple PCR-based molecular marker for bulb color selection in onion (Allium cepa L.) Theor. Appl. Genet. 118: 1391-1399.

Kim S, Binzel M, Yoo K, Park S, Pike LM. 2004a. Inactivation of DFR (Dihydroflavonol 4-reductase) gene transcription results in blockage of anthocyanin production in yellow onions (Allium cepa). Mol. Breed. 14: 253-263.

Kim S, Binzel ML, Yoo K, Park S, Pike LM. 2004b. Pink (P), a new locus responsible for a pink trait in onions (Allium сера) resulting from natural mutations of anthocyanidin synthase. Mol. Genet. Genomics 272: 18-27.

Kim S, Jones R, Yoo K, Pike LM. 2004c. Gold color in onions (Allium cepa): a natural mutation of the chalcone isomerase gene resulting in a pre-mature termination codon. Mol. Genet. Genomics 272: 411-419.

Kim S, Jones R, Yoo K, Pike LM. 2005a. The L locus, one of complementary genes required for anthocyanin production in onions (Allium cepa), encodes anthocyanidin synthase. Theor. Appl. Genet. 111: 120-127.

Kim S, Park JY, Yang T. 2015. Characterization of three active transposable elements recently inserted in three independent $D F R-A$ alleles and one high-copy DNA transposon isolated from the Pink allele of the ANS gene in onion (Allium cepa L.). Mol. Genet. Genomics 290: 1027-1037.
Kim S, Yoo K, Pike LM. 2005b. Development of a PCR-based marker utilizing a deletion mutation in the DFR (dihydroflavonol 4-reductase) gene responsible for the lack of anthocyanin production in yellow onions (Allium cepa). Theor. Appl. Genet. 110: 588-595.

Lotito SB, Frei B. 2006. Comsumption of flavonoids-rich foods and increased plasma antioxidant capacity in humans: cause, consequence, or epiphenomenon? Free Radic. Biol. Med. 41: 1727-1746.

Neu-Yilik G, Gehring NH, Hentze MW, Kulozik AE. 2004. Nonsense-mediated mRNA decay: from vacuum cleaner to Swiss army knife. Genome Biol. 5: 218.

Nishiumi S, Miyamoto S, Kawabata K, Ohnishi K, Mukai R, Murakami A, et al. 2011. Dietary flavonoids as cancerpreventive and therapeutic biofactors. Front. Biosci. 3: 1332-1362.

Petit P, Granier T, d'Estaintot BL, Manigand C, Bathany K, Schmitter J, et al. 2007. Crystal structure of grape dihydroflavonol 4-reductase, a key enzyme in flavonoid biosynthesis. J. Mol. Biol. 368: 1345-1357.

Petroni K, Tonelli C. 2011. Recent advances on the regulation of anthocyanin synthesis in reproductive organs. Plant Sci. 181: 219-229.

Quattrocchio F, Wing JF, Leppen HTC, Mol JN, Koes RE. 1993. Regulatory genes controlling anthocyanin pigmentation are functionally conserved among plant species and have distinct sets of target genes. Plant Cell 5: 1497-1512.

Ramsay NA, Glover BJ. 2005. MYB-bHLH-WD40 protein complex and the evolution of cellular diversity. Trends Plant Sci. 10: 63-70.

Reiman GH. 1931. Genetic factors for pigmentation in the onion and their relation to disease resistance. J. Agr. Res. 42: 251-278.

Rhodes MJC, Price KR. 1996. Analytical problems in the study of flavonoid compounds in onions. Food Chem. 57: 113-117.

Russo M, Spagnuolo C, Tedesco I, Bilotto S, Russo GL. 2012. The flavonoid quercetin in disease prevention and therapy: Facts and fancies. Biochem. Pharmacol. 83: 6-15.

Shaul O. 2015. Unique aspects of plant nonsense-mediated mRNA decay. Trends Plant Sci. 20: 767-779.

Shirley BW. 1996. Flavonoid biosynthesis: 'new' functions for an 'old' pathway. Trends Plant Sci. 1: 377-382. 
Slimestad R, Fossen T, Vågen IM. 2007. Onions: a source of unique dietary flavonoids. J. Agric. Food Chem. 55: 10067-10080.

Song S, Kim C, Moon JS, Kim S. 2014. At least nine independent natural mutations of the $D F R-A$ gene are responsible for appearance of yellow onions (Allium cepa L.) from red progenitors. Mol. Breed. 33: 173-186.

Spelt C, Quattrocchio F, Mol JN, Koes RE. 2000. anthocyanin1 of Petunia encodes a basic helix-loop-helix protein that directly activates transcription of structural anthocyanin genes. Plant Cell 12: 1619-1631.

Veitch NC, Grayer RJ. 2011. Flavonoids and their glycosides, including anthocyanins. Nat. Prod. Rep. 28: 1626-1695.

Yamazaki M, Makita Y, Springob K, Saito K. 2003. Regulatory mechanisms for anthocyanin biosynthesis in chemotypes of Perilla frutescens var. crispa. Biochem. Eng. J. 14: 191-197. 